\title{
Loss of ELK1 has differential effects on age-dependent organ fibrosis
}

Jennifer T. Cairns ${ }^{\mathrm{a}}$, Anthony Habgood ${ }^{\mathrm{a}}$, Rochelle C. Edwards-Pritchard ${ }^{\mathrm{a}}$, Chitra Joseph ${ }^{\mathrm{a}}$, Alison E. John ${ }^{\mathrm{a}}$, Chloe Wilkinson ${ }^{\mathrm{a}}$, Iain D. Stewart ${ }^{\mathrm{a}}$, Jack Leslie ${ }^{\mathrm{b}}$, Burns C. Blaxall ${ }^{\mathrm{c}}$, Katalin Susztak $^{\mathrm{d}}$, Siegfried Alberti ${ }^{\mathrm{e}}$, Alfred Nordheim ${ }^{\mathrm{e}, \mathrm{f}}$, Fiona Oakley ${ }^{\mathrm{b}}$, Gisli Jenkins ${ }^{\mathrm{a}}$, Amanda L. Tatler ${ }^{\mathrm{a}, *}$

${ }^{a}$ Nottingham NIHR Biomedical Research Centre, Division of Respiratory Medicine, University of Nottingham, Nottingham University Hospitals, City Campus, Nottingham, NG5 1PB, UK

${ }^{\mathrm{b}}$ Newcastle Fibrosis Research Group, Institute of Cellular Medicine, Faculty of Medical Sciences, 4th Floor, William Leech Building, Newcastle University, Framlington Place, Newcastle upon Tyne, NE2 4HH, UK

${ }^{\mathrm{c}}$ Department of Personalized Medicine and Pharmacogenetics, The Christ Hospital Health Network, Cincinnati, OH, USA

${ }^{\mathrm{d}}$ Renal Electrolyte and Hypertension Division, Department of Medicine, Department of Genetics, University of Pennsylvania, Perelman School of Medicine, Philadelphia, PA, USA

${ }^{\mathrm{e}}$ Interfaculty Institute of Cell Biology, Tuebingen University, Tuebingen, Germany

${ }^{\mathrm{f}}$ Leibniz Institute on Ageing (FLI), Jena, Germany

A R T I C L E I N F O

\section{Keywords:}

Fibrosis

ELK1

Ageing

TGF $\beta$

Integrin

Lung

Liver

Gene regulation

CSE

\begin{abstract}
A B S T R A C T
ETS domain-containing protein-1 (ELK1) is a transcription factor important in regulating $\alpha v \beta 6$ integrin expression. $\alpha v \beta 6$ integrins activate the profibrotic cytokine Transforming Growth Factor $\beta 1$ (TGF $\beta 1$ ) and are increased in the alveolar epithelium in idiopathic pulmonary fibrosis (IPF). IPF is a disease associated with aging and therefore we hypothesised that aged animals lacking Elk1 globally would develop spontaneous fibrosis in organs where $\alpha \mathrm{v} \beta 6$ mediated TGF $\beta$ activation has been implicated. Here we identify that Elk1-knockout $\left(E l k 1^{-\prime}\right.$ ${ }^{o}$ ) mice aged to one year developed spontaneous fibrosis in the absence of injury in both the lung and the liver but not in the heart or kidneys. The lungs of $E l k 1^{-10}$ aged mice demonstrated increased collagen deposition, in particular collagen $3 \alpha 1$, located in small fibrotic foci and thickened alveolar walls. Despite the liver having relatively low global levels of ELK1 expression, $E l k 1^{-/ O}$ animals developed hepatosteatosis and fibrosis. The loss of Elk1 also had differential effects on Itgb1, Itgb5 and Itgb6 expression in the four organs potentially explaining the phenotypic differences in these organs. To understand the potential causes of reduced ELK1 in human disease we exposed human lung epithelial cells and murine lung slices to cigarette smoke extract, which lead to reduced ELK1 expression andmay explain the loss of ELK1 in human disease. These data support a fundamental role for ELK1 in protecting against the development of progressive fibrosis via transcriptional regulation of beta integrin subunit genes, and demonstrate that loss of ELK1 can be caused by cigarette smoke.
\end{abstract}

\section{Introduction}

Tissue fibrosis is a common pathogenic pathway that results in a range of pathologies across several organs including the lung, liver, skin, kidney and heart. Fibrosis of these tissues is responsible for more than a third of deaths throughout the world (Wynn, 2008). There are shared, and distinct, mechanisms that may lead to fibrosis in a number of organs involving a range of cells which can provoke inflammation, epithelial injury and fibroproliferation (Wynn, 2008; Weiskirchen et al., 2019).

A key pathway promoting fibrosis involves integrin-mediated TGF $\beta$ activation. Integrin-mediated TGF $\beta$ activation has been shown to be important in fibrosis of the lung, liver, skin, cardiovascular system and kidney (Henderson et al., 2013; Tatler and Jenkins, 2012; Popov et al., 2008; Häkkinen et al., 2004; Hahm et al., 2007), with avß6 integrinmediated TGF $\beta$ activation being particularly important in the lung (Tatler and Jenkins, 2012) while $\alpha v \beta 1$ integrin-mediated TGF $\beta$ activation being considered more important for liver and kidney fibrosis (Reed et al., 2015; Chang et al., 2017).

ELK1 is an X-linked transcription factor that regulates a range of biological processes (Sharrocks, 2001). ELK1 is ubiquitously expressed in a range of cell types and in a number of organs (Uhlén et al., 2015).

\footnotetext{
* Corresponding author at: Division of Respiratory Medicine, B11 Clinical Sciences Building, Nottingham City Hospital, Hucknall Road, Nottingham, NG5 1PB, UK.

E-mail address: amanda.tatler@nottingham.ac.uk (A.L. Tatler).
} 
We have previously demonstrated that ELK1 is a transcriptional repressor that prevents excessive upregulation of the $\beta 6$ integrin subunit following injury (Tatler et al., 2016). Furthermore, we have shown that loss of ELK1 leads to enhanced pulmonary fibrosis following bleomycininduced lung injury in mice and that patients with Idiopathic Pulmonary Fibrosis (IPF) have reduced expression of ELK1 in their lungs (Tatler et al., 2016).

Idiopathic fibrotic disease of many organs is associated with aging. Therefore, we hypothesized that loss of ELK1 would lead to age-related tissue fibrosis of internal organs due to loss of repressed integrin expression. To test this hypothesis $E l k 1$-knockout $\left(E l k 1^{-10}\right)$ mice were allowed to age to one year after which their internal organs were assessed for fibrosis. We demonstrated that the lungs and liver from $E L K 1^{-/ O}$ mice developed spontaneous age-related fibrosis whereas the heart and kidneys were normal. To understand the potential mechanism by which ELK1 may be lost in IPF, we exposed human lung epithelial cells and murine lung slices to cigarette smoke extract (CSE) and confirmed that this reduced ELK1 protein levels. These studies illustrate the importance of ELK1 in protection against tissue fibrosis and highlight a mechanism through which this protective mechanism may be lost in fibrosis.

\section{Methods}

\subsection{Cell lines and reagents}

Immortalised human bronchial epithelial cells (iHBECs; courtesy Dr Shay, University of Texas) were used for cigarette smoke extract (CSE) experiments. iHBECs were cultured in keratinocyte-serum free medium (K-SFM; Gibco, UK) supplemented with $25 \mu \mathrm{g} / \mathrm{ml}$ bovine pituitary extract (Gibco, UK), $0.2 \mathrm{ng} / \mathrm{ml}$ epidermal growth factor (Gibco, UK), 25 $\mu \mathrm{g} / \mathrm{ml} \mathrm{G418} \mathrm{sulphate} \mathrm{(VWR} \mathrm{Life} \mathrm{Science,} \mathrm{UK)} \mathrm{and} 250 \mathrm{ng} / \mathrm{ml}$ puromycin (MilliporeSigma, UK). ELK1 \#ab32106, ß-tubulin \#ab6046, GAPDH \#ab181602, aSMA \#ab5694 and NIMP \#ab2557 antibodies for western blotting and/or immunohistochemistry (IHC) were from Abcam, UK. CD3 + \#MCA1477 and CD68 \#OABB00472 antibodies for IHC were obtained from Bio-rad, UK and Aviva Systems Biology, UK, respectively. Reagents required for the synthesis of cDNA from RNA, were supplied from Invitrogen, UK (SuperScript ${ }^{\mathrm{TM}}$ IV Reverse Transcriptase), Qiagen, UK (Nuclease free water), Roche, UK (OligoDT) or Promega, UK (RNasin inhibitor, dNTPs). Tobacco laboratory research grade cigarettes (batch 1R6F) were from the University of Kentucky, USA.

\subsection{Invivo studies}

All animal care and procedures were approved by the University of Nottingham Ethical Review Committee and were performed under Home Office Project and Personal License authority within the Animal (Scientific Procedures) Act 1986. Animals were housed in the Biomedical Services Unit, University of Nottingham. Male Elk1knockout $\left(E l k 1^{-/ 0}\right)$ (Cesari et al., 2004) and wildtype $\left(E l k 1^{+/ 0}\right)$ mice were aged for 12 or 52 weeks with free access to food (Tekland Global $18 \%$ protein rodent diet, UK) and water. Lungs, liver, kidneys and heart were either removed and snap-frozen in liquid nitrogen and then stored at $-80{ }^{\circ} \mathrm{C}$ for mRNA and hydroxyproline assessment, or insufflated with $10 \%$ formalin (VWR chemicals, UK) at constant gravitational pressure $\left(20 \mathrm{~cm} \mathrm{H}_{2} \mathrm{O}\right)$ then paraffin wax embedded for histology and immunohistochemistry.

\subsection{Reverse transcription and quantitative polymerase chain reaction $(q P C R)$}

Murine lung, liver, kidney and heart tissue was ground into powder in liquid nitrogen and RNA extracted using TRIzol using standard methods. Alternatively, RNA from iHBECs were prepared using a
NucleoSpin RNA II kit (Macherey-Nagel, UK) according to manufacturer's instructions. For detailed methods see supplementary methods.

\subsection{SDS-PAGE, western blot}

Ground murine lung, liver, kidney and heart tissue as well as Precision Cut Lung Slices (PCLS) and iHBECs were lysed in whole cell lysis buffer (Cell Signaling, UK) supplemented with protease and phosphatase inhibitors (Complete Mini protease inhibitor tablet and Phos-stop inhibitor tablets; Roche, UK) and processed for immunoblotting using standard methods see supplementary methods

\subsection{Histological assessment}

Histological sections of murine lung, liver and heart tissue, were cut to between 3-5 microns and dewaxed in xylene prior to rehydration in decreasing concentrations of ethanol.

The sections were incubated in either Mayer's haemotoxylin, and eosin, Weigert's haemotoxylin and Sirius red or Masson's Trichrome prior to dehydration and mounting. Tissue staining was imaged using Nikon Eclipse 90i microscope and NIS Elements AR3.2 software (Nikon). Heart tissue sections were scanned using a Hammamatsu photonics K. K. C9600-02 and selected and captured using Hamamatsu NDP.view2 software.

\subsection{Immunohistochemistry}

Tissue sections were also subjected to immunohistochemistry using standard methods see supplementary methods.

\subsection{Assessment of tissue fibrosis}

The following scores were assigned to describe the severity of fibrosis in Sirius red stained liver sections; $0=$ normal, $0.5=$ very mild fibrosis, $1=$ mild fibrosis, $2=$ fibrosis. The following scores were assigned to assess the grade of steatosis in H\&E stained liver sections; $0=$ $0-5 \%$ steatosis, $1=5-33 \%, 2=33-66 \%$ and $3=>66 \%$ based on the Brunt score (Brunt et al., 1999). Quantification of alveolar wall thickness in lungs of $E l k 1^{-/ O}$ and $E l k 1^{+/ O}$ mice was performed as follows. 10 random fields of view were observed per tissue section. Each field of view was overlaid with a graticule. The alveolar wall thickness was measured each time it intercepted a horizontal line. The mean value in $\mu \mathrm{m}$ was calculated for each mouse. All scoring was undertaken blinded to the underlying genotype.

Biochemical assessment of tissue fibrosis was performed by measuring hydroxyproline in murine lung tissue as previously described (Uhlén et al., 2015).

\subsection{Precision cut lung slices (PCLS)}

6-week-old mice were euthanised and via a cannula to the trachea, their lungs were slowly inflated using $1.3 \mathrm{ml} 2 \%$ low melting point agarose in PBS followed by $0.2 \mathrm{ml}$ air. Lungs were removed and placed in ice cold DMEM supplemented with $4 \%$ L-Glutamine, $4 \%$ Penicillin/ Streptomycin and $2 \%$ Amphotericin B (Gibco, UK). Lung lobes were separated and sliced in chilled HBSS buffer at a thickness of $250 \mu \mathrm{m}$ using the VT1200S Vibratome (Leica) set to $1 \mathrm{~mm}$ amplitude, $1 \mathrm{~mm}$ / second speed. The slices were then placed in warm supplemented DMEM media and incubated overnight at $37{ }^{\circ} \mathrm{C}, 5 \% \mathrm{CO}_{2}$. The slices were transferred to freezing media (FBS with $10 \%$ DMSO) and stored long-term in liquid nitrogen. PCLS were recovered by rapidly thawing frozen vials at $37{ }^{\circ} \mathrm{C}$ and transferring them to fresh supplemented DMEM for a period of $24 \mathrm{~h}$ at $37{ }^{\circ} \mathrm{C}, 5 \% \mathrm{CO}_{2}$ before use in subsequent experiments. 3 PCLS were used per condition in CSE experiments. 


\subsection{Preparation of CSE and experimental design}

Cigarette Smoke Extract (CSE) was generated by smoking two tobacco laboratory research grade cigarettes (batch 1R6F) into $20 \mathrm{ml}$ of K-SFM media (iHBEC) or DMEM (PCLS) using a vacuum pump under a constant pressure of 0.2 bar. Media was filter-sterilized and absorbance was measured at $320 \mathrm{~nm}$. Prepared CSE was aliquoted and stored at $-20{ }^{\circ} \mathrm{C}$ until required. From this 1, 2, 3, 4 and $5 \%$ CSE were prepared in K-SFM for iHBECs or DMEM (3 \% CSE only) for PCLS. CSE was prepared in this manner daily and applied for 7 days.

\subsection{Statistics}

All data are reported as mean \pm standard deviation (parametric) or median (non-parametric) of $\mathrm{n}$ observations. Statistical significance for parametric data was determined by either an unpaired $t$-test when comparing two data fields or ANOVA for comparing multiple data fields. Data that were not normally distributed were assessed by nonparametric Mann-Whitney test to determine significance. CSE experiments were repeated three times and expressed as mean data from the three independent experiments. Statistical analysis of QPCR experiments were performed using an unpaired Welch's $t$-test. P values less than 0.05 were considered significant. All statistical analysis was performed using GraphPad Prism (v7.04, La Jolla, CA, USA).

\section{Results}

\subsection{ELK1 is expressed in multiple internal organs}

To determine the importance of baseline ELK1 expression in agerelated fibrosis, internal organs were harvested from mice at 1 year of age followed by assessment of Elk1 mRNA and protein. Elk1 mRNA (Fig. 1A) and ELK1 protein (Fig. 1B and C) were expressed at the highest levels in the lung and heart tissue. In contrast, levels of Elk1 mRNA and protein were expressed at the lowest levels in the liver.

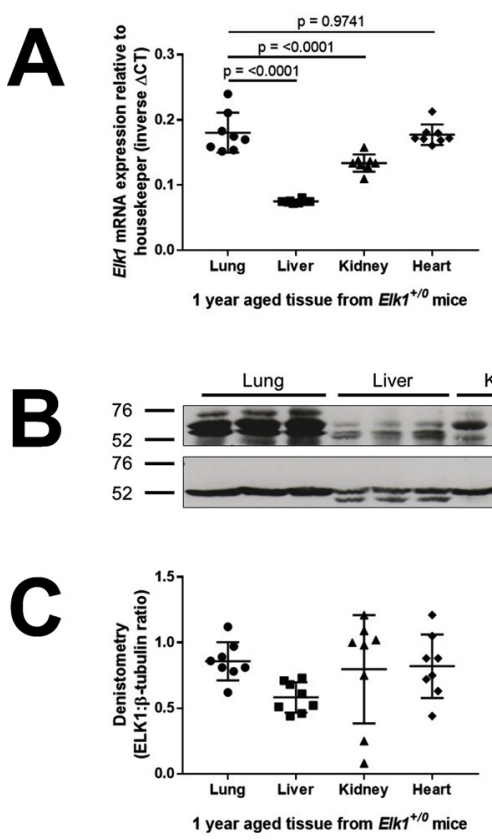

Fig. 1. ELK1 expression in internal organs in wild-type animals.

(A) Elk1 mRNA expression in the lung, liver, kidney and heart tissues of 1-year aged $E l k 1^{+/ O}$ mice. $\mathrm{n}=8$, P values determined by one-way ANOVA.

(B) Representative western blot showing ELK1 protein expression in the lung, liver, kidney and heart tissues of 1 -year aged $E l k 1^{+/ 0}$ mice.

(C) Densitometry analysis showing the ratio of ELK1: $\beta$-tubulin. $\mathrm{n}=8$ mice.
Levels of ELK1 in the kidneys were generally lower than the heart and lungs but greater than observed in the liver (Fig. 1B and C).

\subsection{Elk $1^{-/ O}$ mice develop age related pulmonary fibrosis}

The lungs from 1-year old $E l k 1^{-/ O}$ mice were assessed for evidence of pulmonary fibrosis. Despite not being subjected to injury, hydroxyproline levels were mildly elevated in the lungs of $E l k 1^{-/ O}$ animals compared with controls (Fig. 2A). The elevated collagen levels in the lungs of $E l k 1^{-10}$ mice were reflected in the lung architecture with features consistent with spontaneous lung fibrosis including small foci of alveolar destruction with increased collagen deposition and areas of lymphoid aggregates (Supplemental Fig. 1A). Analysis of these intermittent small fibrotic lesions with Sirius red staining using polarised light microscopy revealed that their composition was predominantly type I collagen (yellow/orange strand) and type III collagens (green strands) (Fig. 2B). This was confirmed using immunohistochemistry to demonstrate that both type I and type III collagens are increased within the lungs of aged Elk1 $1^{-/ 0}$ animals compared with controls (Fig. 2C). Furthermore, the alveolar walls of $E l k 1^{-/ O}$ mice were thicker compared with wild-type control lungs (Fig. 2D and E). Sirius Red staining revealed that there was increased deposition of collagen III within the alveolar interstitium (Fig. 2E). Analysis of collagen type 1, 3 and 6 mRNA expression (Fig. 2F) did not show any difference in Col1a1 and Col6a1 mRNA expression between $E l k 1^{+/ O}$ and $E l k 1^{-/ O}$ animals. However, Col3a1 expression was significantly increased in $E l k 1^{-/ 0}$ mice consistent with the increased interstitial collagen 3 seen histologically (Fig. 2E). Interestingly, the phenomenon of thickened alveolar walls, deposition of fibrosis and increased Col3a1 mRNA expression was not observed in 12-week old $E l k 1^{-/ O}$ mice (Supplemental Fig. 2A and 2B), highlighting the importance of ageing in the development of this phenotype in $E l k 1^{-10}$ mice. While inflammation is not thought to be a major pathogenic pathway in IPF, inflammation is associated with some forms of pulmonary fibrosis and so we investigated the presence of activated T cells, neutrophils and macrophages in the lungs of the aged animals. We found no difference in numbers of any of these cell types between the lungs of aged Elk1 $1^{-/ 0}$ and Elk1 $1^{+/ 0}$ animals (Fig. $2 \mathrm{G}$ and Supplemental Fig. 1B).

\subsection{Elk $1^{-/ 0}$ mice do not develop age related cardiac or renal fibrosis}

As described earlier, ELK1 is expressed at high levels in the normal heart, and to a lesser extent in the kidneys. Therefore, the heart and kidneys were assessed for phenotypic abnormalities. There was no evidence of fibrosis in the heart tissue of $E l k 1^{+/ 0}$ and $E l k 1^{-/ O}$ animals as determined either histologically or biochemically. Indeed, there was a slight reduction of collagen in the hearts of $E l k 1^{-/ O}$ animals compared with controls (Fig. 3A), although morphological assessment of heart sections using Sirius Red showed no abnormalities in tissue structure in either wild-type or knock-out animals (Fig. 3B). In the kidneys of $E l k 1^{+/ O}$ and $E l k 1^{-/ O}$ mice, there was no evidence of renal fibrosis biochemically (Fig. 3C) or histologically (Fig. 3D).

\subsection{Elk $1^{-/ O}$ mice develop hepatosteatosis}

Although high levels of ELK1 were not observed in whole liver lysates at either the mRNA or protein level in $E l k 1^{+/ 0}$ mice, there was evidence of pathology on histology, in the livers of aged $E l k 1^{-/ O}$ mice. Liver sections from $E l k 1^{+/ O}$ and $E l k 1^{-/ O}$ aged mice, stained with Sirius Red, demonstrated increased portal fibrosis and mild perisinusoidal fibrosis in the $E l k 1^{-/ O}$ animals compared with $E l k 1^{+/ O}$ mice. In addition, Elk1 $1^{-10}$ mice also exhibited more micro- and macrosteatosis shown by the blue and green arrows, respectively (Fig. 4A and B). However, there was no increase in hydroxyproline levels in the livers of Elk1 $1^{-/ O}$ mice (Supplementary Fig. 3).

To further explore the fibrotic phenotype in the liver, 


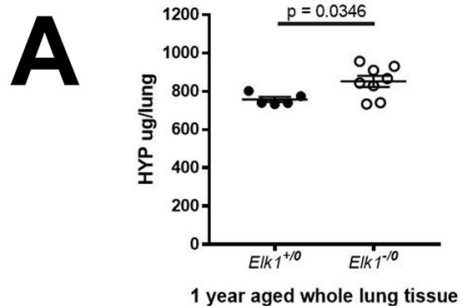

1 year aged whole lung tissue
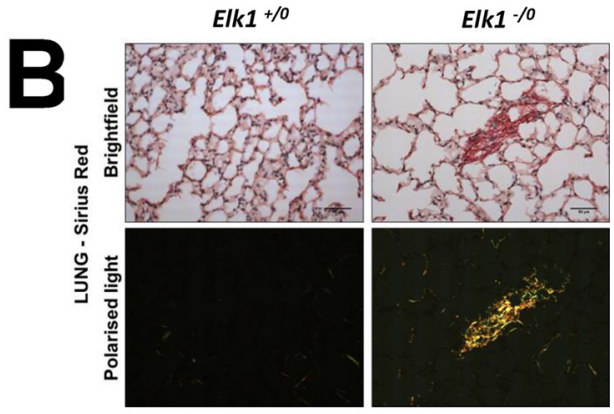

C
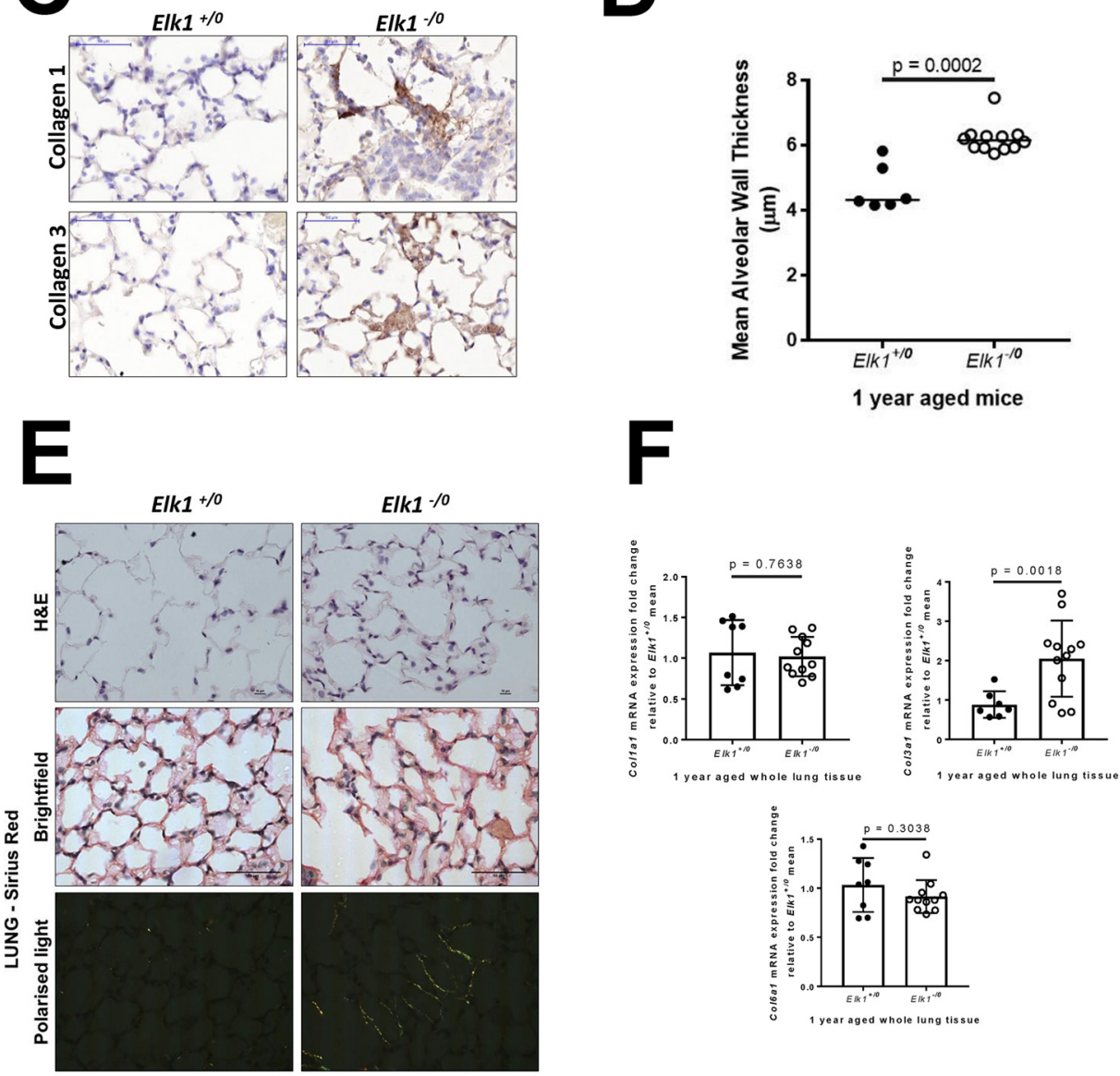

1 year aged mice

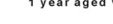

Fig. 2. $E l k 1^{-/ O}$ aged mice deposit collagen and exhibit small fibrotic lesions in the lungs.

(A) Total lung collagen assessed by measuring hydroxyproline levels in ground lung tissue from 1 -year aged $E l k 1^{+/ O}$ and $E l k 1^{-/ O}$ mice. $\mathrm{P}$ value determined by unpaired $t$-test.

(B) Representative brightfield and polarised light images of Sirius Red stained lung sections from 1-year aged $E l k 1^{+/ O}$ and $E l k 1^{-/ O}$ mice. Scale bar $50 \mu \mathrm{m}$.

(C) Representative images of lung sections from 1-year aged Elk $1^{+/ O}$ and $E l k 1^{-/ O}$ mice probed for Collagen type I and Collagen type III. P value determined by unpaired $t$-test. Scale bar $50 \mu \mathrm{m}$.

(D) Quantification of alveolar wall thickness in 1 -year aged $E l k 1^{+/ O}$ and $E l k 1^{-/ O}$ mice. P value determined by Mann-Whitney test.

(E) Representative brightfield and polarised light images of H\&E and Sirius Red stained lung sections from 1-year aged Elk1 $1^{+/ O}$ and $E l k 1^{-/ O}$ mice. Scale bar $50 \mu \mathrm{m}$.

(F) Col1a1, Col3a1 and Col6a1 mRNA expression in $E l k 1^{+/ O}$ and $E l k 1^{-/ O}$ in 1-year aged mice were analysed using QPCR. Data were expressed as mRNA expression fold-change relative to $E l k 1^{+/ O}$ mean of 1 . P value determined by $t$-test.

(G)Quantification of cell numbers of CD3 (activated T-cells), NIMP (neutrophils) and CD68 (macrophages) positive cells in lung sections from 1-year aged $E l k 1^{+/ O}$ and $E l k 1^{-/ O}$ mice.). P value determined by unpaired $t$-test.
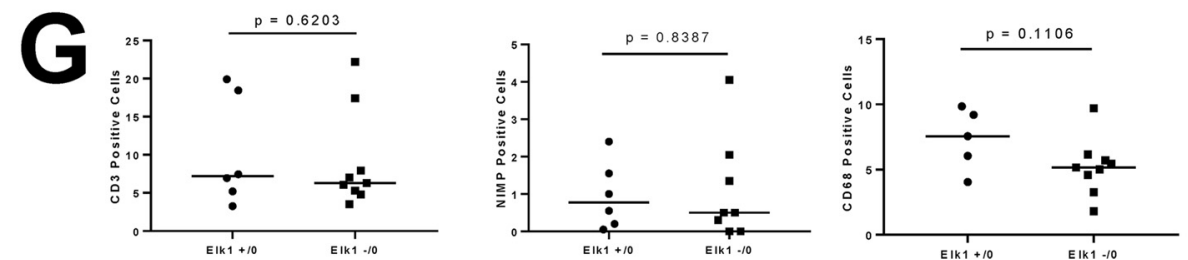

immunohistochemistry (IHC) was performed using a marker of hepatic myofibroblasts. This demonstrated that alpha Smooth Muscle Actin (aSMA) was not expressed in the livers of $E l k 1^{+/ 0}$, however aSMA staining was increased in the livers from $E l k 1^{-/ O}$ aged animals and localised to the peri-portal and peri-sinusoidal regions (Fig. 4C).

In contrast with pulmonary fibrosis, liver fibrosis is more obviously associated with inflammation, therefore sections were assessed for the presence of inflammatory cells such as; activated T-cells (CD3), neutrophils (NIMP) as well as monocyte and macrophage populations
(CD68) (Fig. 4D). Quantification of the inflammatory cell populations revealed a slight increase in the mean number of activated T-cells and neutrophils in $E l k 1^{-/ O}$ mice although not statistically different. However, there was a substantial, and statistically significant, increase in the number of macrophages present in the livers from $E l k 1^{-/ O}$ aged animals compared with wild-type controls (Fig. 4D). Despite the obvious fibrotic phenotype within the lungs and livers of aged Elk1 $1^{-/ 0}$ animals, and the presence of fat within the livers, we observed no difference in animal body mass at 1 year between the two genotypes 
A

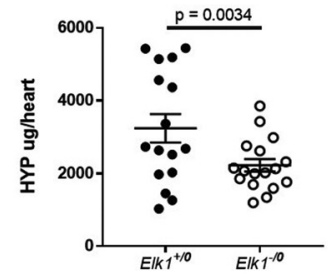

1 year aged whole heart tissue

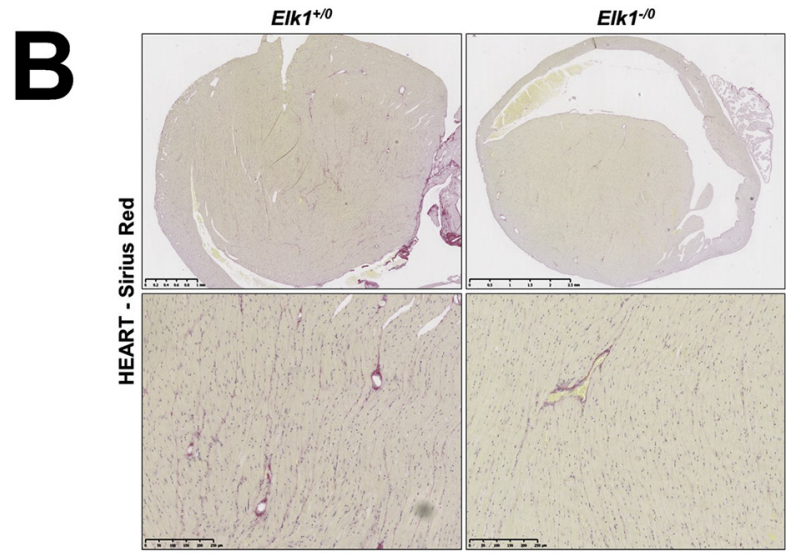

C

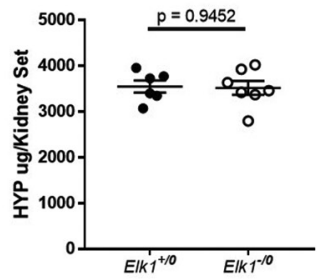

1 year aged whole kidney tissue

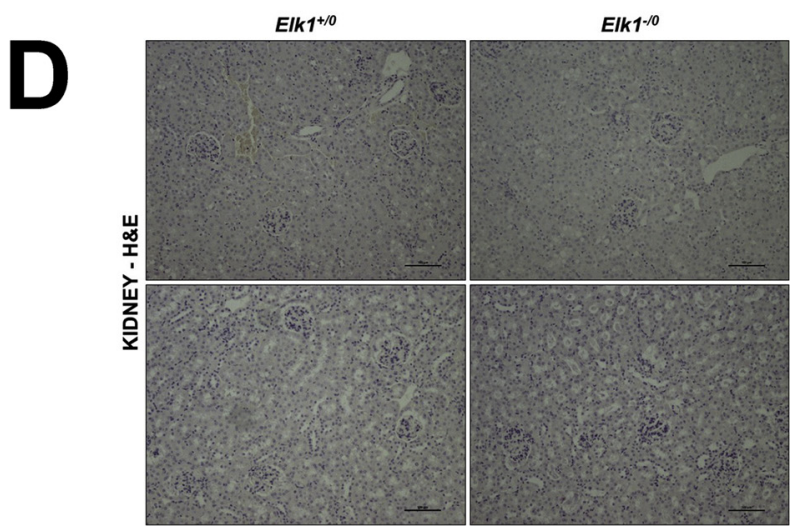

Fig. 3. Elk $1^{-/ 0}$ aged mice do not display any pathological abnormalities in the kidney or heart.

(A)Total heart collagen assessed by measuring hydroxyproline levels in ground heart tissue from 1-year aged $E l k 1^{+/ O}$ and $E l k 1^{-/ O}$ mice. P value determined by unpaired $t$-test.

(B) Representative images of Sirius Red stained heart sections from 1-year aged Elk $1^{-10}$ and $E l k 1^{+/ O}$ mice.

(C)Total kidney collagen assessed by measuring hydroxyproline levels in ground kidney tissue from 1-year aged $E l k 1^{+/ 0}$ and $E l k 1^{-/ O}$ mice. P value determined by unpaired $t$-test.

(D)Representative images of H\&E stained kidney sections from 1-year aged $E l k 1^{-/ O}$ and $E l k 1^{+/ o}$ mice.
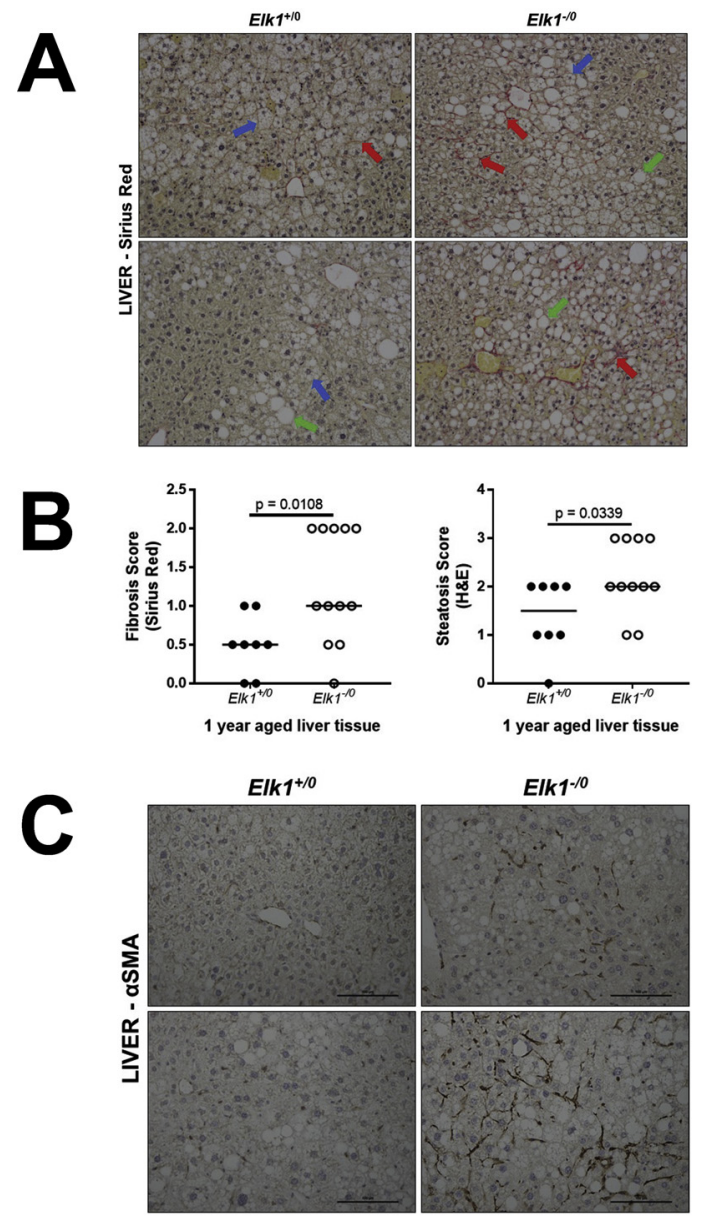

Elk 1/0
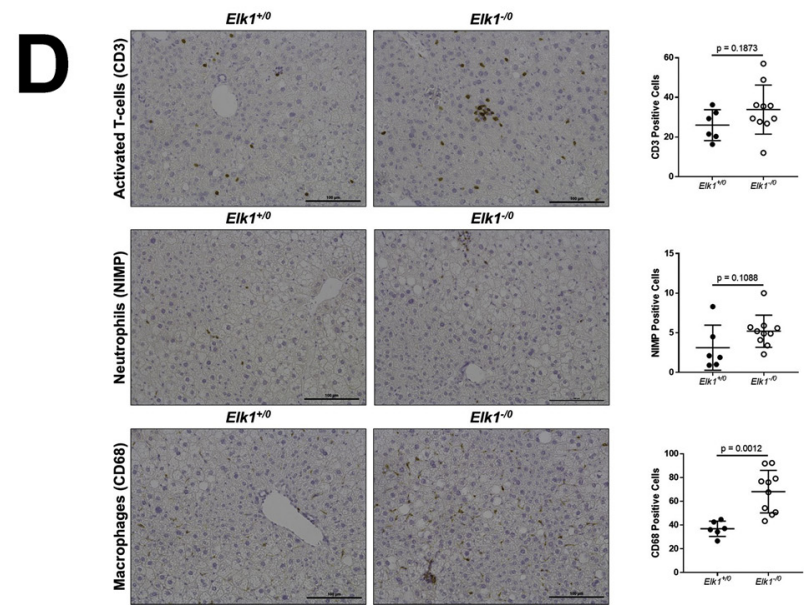

Fig. 4. $E l k 1^{-/ O}$ aged mice have mild hepatic fibrosis and steatosis in the absence of injury.

(A) Representative images of Sirius Red stained liver sections from 1-year aged $E l k 1^{+/ O}$ and $E l k 1^{-/ O}$ mice showing mild fibrosis (red arrow), micro-steatosis (blue arrows) and macro-steatosis (green arrows).

(B) H\&E and Sirius Red stained liver sections from 1-year aged $E l k 1^{+/ 0}$ and $E l k 1^{-/ O}$ mice scored blind for fibrosis and steatosis. P values determined by Mann-Whitney test.

(C) Representative images of liver sections from 1-year aged $E l k 1^{+/ 0}$ and Elk $1^{-10}$ mice assessed for $\alpha$ SMA.

(D) Representative images of liver sections from 1-year aged $E l k 1^{+/ 0}$ and Elk $1^{-/ 0}$ mice probed for CD3 (activated T-cells), NIMP (neutrophils) and CD68 (macrophages). $\mathrm{P}$ value determined by unpaired $t$-test. 

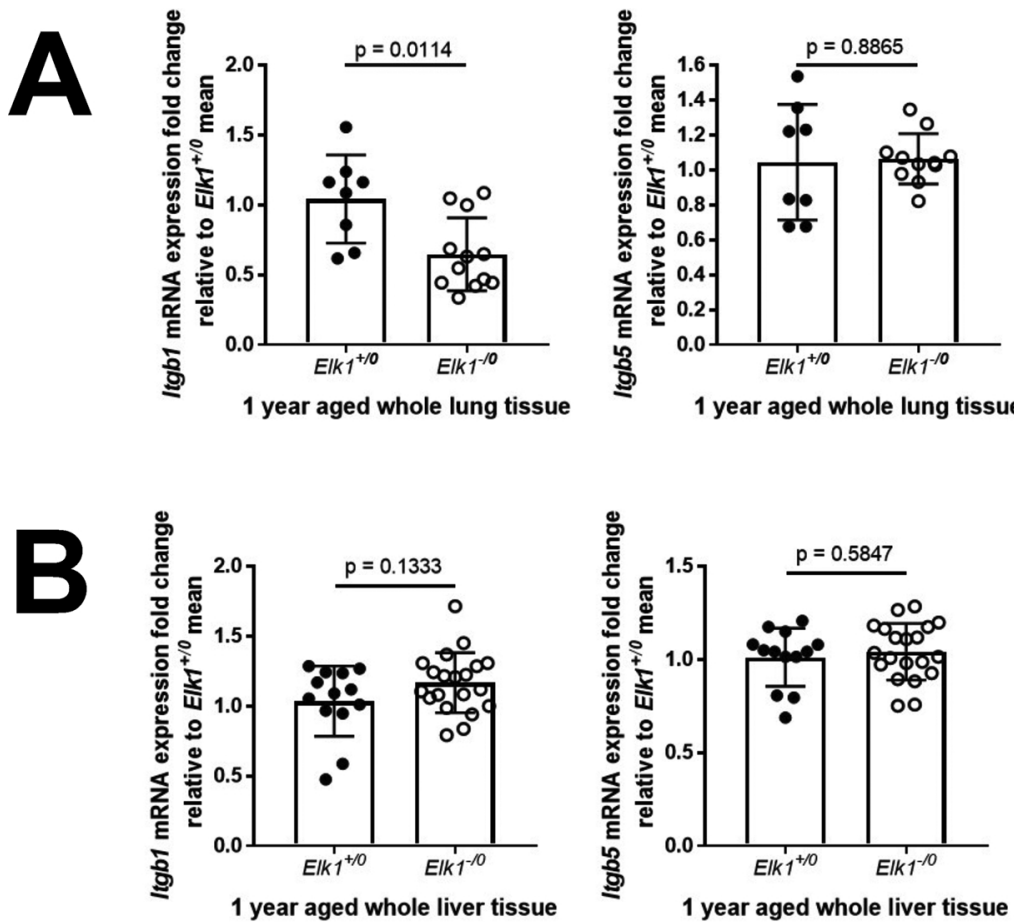

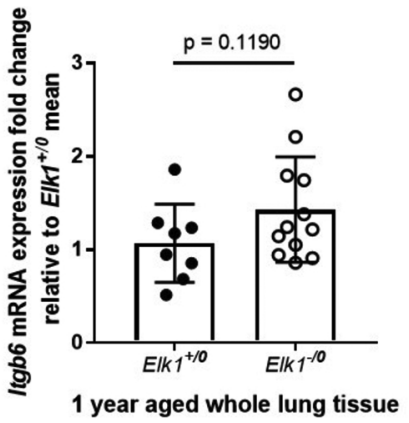

1 year aged whole lung tissue 1 year aged whole lung tissue
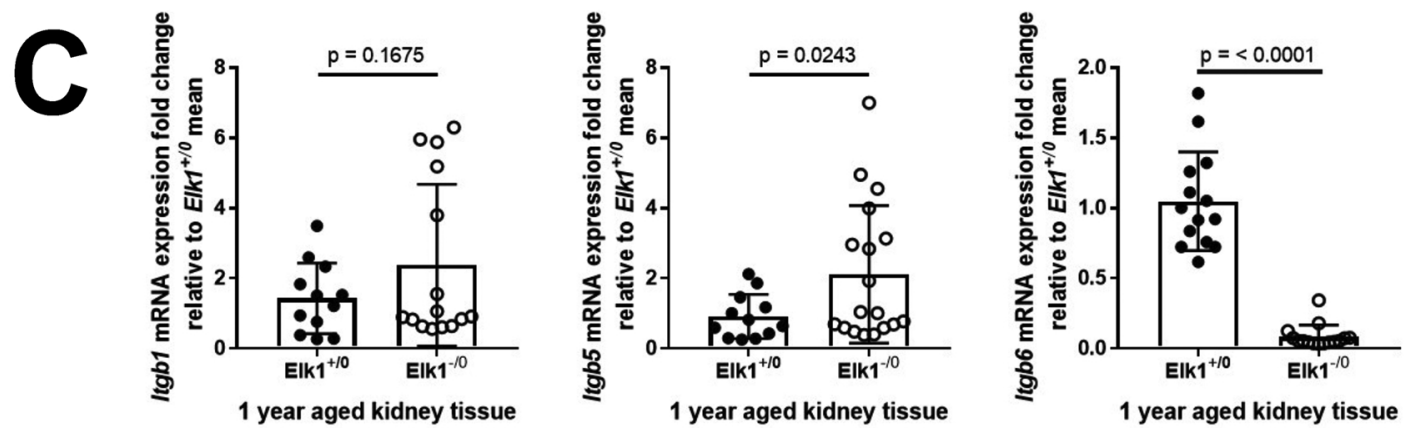

Fig. 5. Loss of ELK1 has organ-specific effects on integrin transcription.

(A)Itgb1, Itgb5 and Itgb6 mRNA expression in the lungs of 1-year aged $E l k 1^{+/ 0}$ and $E l k 1^{-/ 0}$ mice. Data were expressed as mRNA expression fold-change relative to $E l k 1^{+/ 0}$. P values were determined by Welch's $t$-test.

(B) Itgb1 and Itgb5 mRNA expression in the livers of 1-year aged $E l k 1^{+/ 0}$ and $E l k 1^{-/ 0}$ mice were analysed using QPCR. Data were expressed as mRNA expression foldchange relative to $E l k 1^{+/ 0}$. P values were determined by Welch's $t$-test.

(C)Itgb1, Itgb5 and Itgb6 mRNA expression in the kidneys of 1-year aged $E l k 1^{+/ 0}$ and $E l k 1^{-10}$ mice were analysed using QPCR. Data were expressed as mRNA expression fold-change relative to $E l k 1^{+/ O}$. P values were determined by Welch's $t$-test.

(Supplemental Fig. 4).

\subsection{Loss of Elk1 differentially affects integrin expression}

To understand the phenotype of aged Elk1 mice we assessed mRNA levels of key pro-fibrotic integrins in the lungs, liver and kidneys of $E l k 1^{-/ O}$ and $E l k 1^{+/ O}$ animals. In the lungs of $E l k 1^{-/ O}$ mice a significant decrease in Itgb1 mRNA was seen compared with controls animals, whereas Itgb5 levels were unchanged between $E l k^{+/ 0}$ and $E l k 1^{-10}$ animals. There was 1.5 fold increase in Itgb6 was observed in the lungs of $E l k 1^{-/ O}$ mice compared with $E l k 1^{+/ 0}$ mice although this did not reach statistical significance (Fig. 5A).

Levels of Itgb6 were below the detection limits in the livers of both $E l k^{+/ 0}$ and $E l k 1^{-/ O}$ animals (data not shown) and neither whole organ expression of Itgb1 nor Itgb5 mRNA expression were different between Elk $1^{-/ O}$ mice and control animals (Fig. 5B).

The kidneys, however, showed a marked reduction in Itgb6 mRNA levels in $E l k 1^{-/ 0}$ mice and conversely approximately a two fold increase in Itgb5 whicxh was statistically significant and Itgb1 levels which did not reach statistical significance (Fig. 5C).

\subsection{Loss of ELK1 can be acquired following inhalation injury}

We have previously shown that $E l k 1^{-/ O}$ mice exhibit an exaggerated fibrotic response to bleomycin induced fibrosis compared with $E l k 1^{+/ o}$ controls and that IPF patients have lower levels of Elk1 protein in their lungs (Uhlén et al., 2015). We investigated whether ageing caused a decrease in Elk1 expression in the 1-year old Elk1 + /0 mice compared with 12-week-old Elk1 + /0 mice and found no difference in expression (Supplementary Fig. 5). Genetic studies have not identified an association with loss of function Elk1 mutants and pulmonary fibrosis, and it remains unclear whether ELK1 levels can be altered following injury. Therefore, the effect of prolonged exposure to a known fibrogenic risk factor in human disease, cigarette smoke extract (CSE), on epithelial ELK1 expression was assessed.

Levels of Elk1 mRNA in iHBECs changed in a time and concentration 

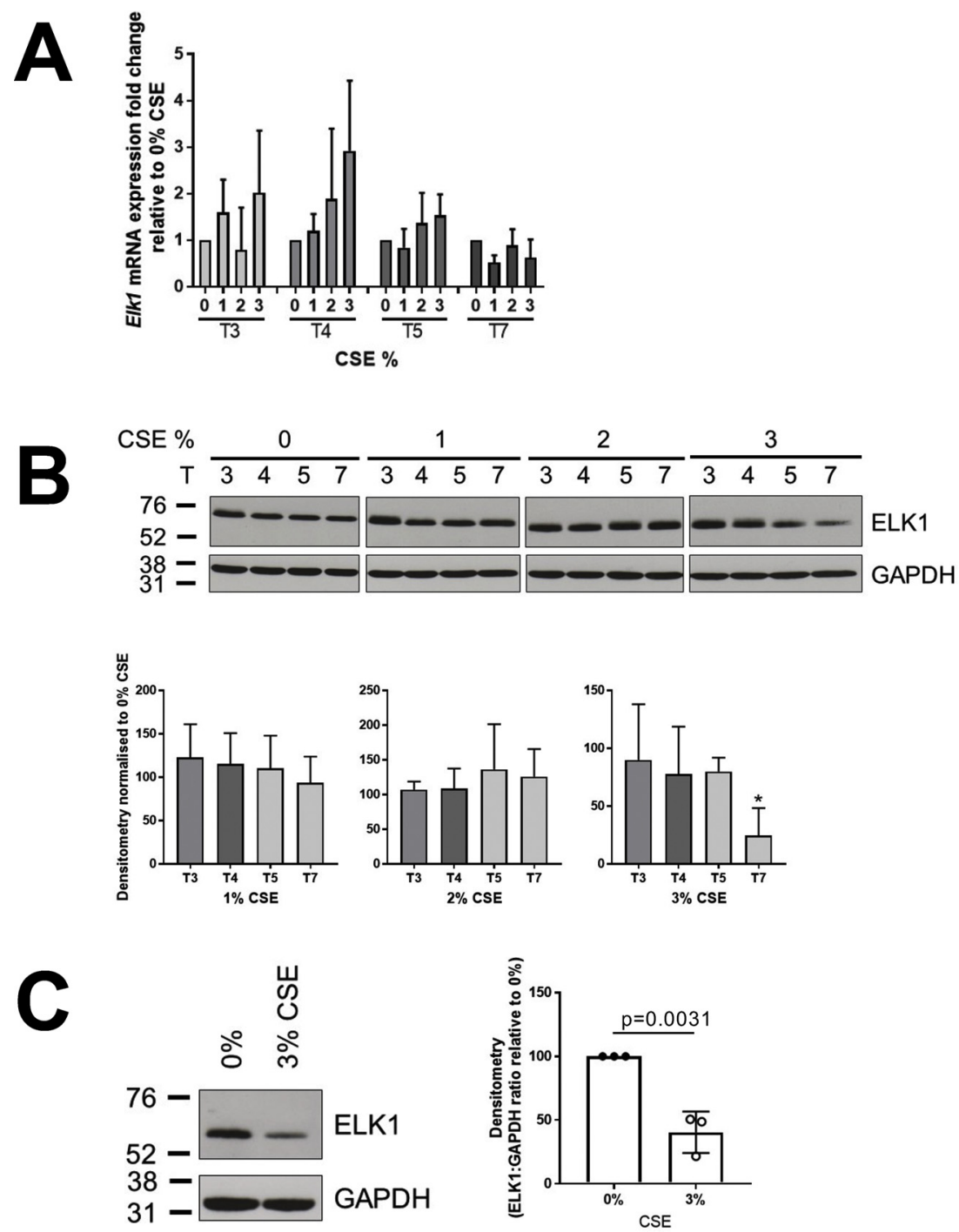

Fig. 6. Prolonged exposure to CSE in the lungs results in the loss of ELK1.

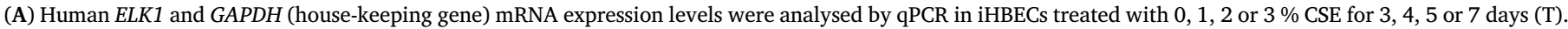

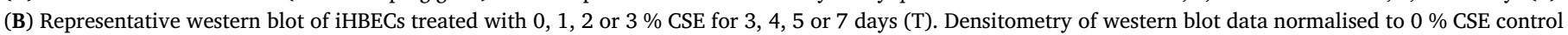
for equivalent time point. $\mathrm{P}$ values determined by one-way ANOVA, $0 \%$ vs $3 \%$ CSE at T7 $(n=3)$.

(C) Representative western blot showing protein expression of ELK1 in PCLS treated with $3 \%$ CSE for 7 days. N = 3 .

dependent manner following culture in CSE, with early time-points demonstrating an increase in Elk1 particularly at higher concentrations, whereas, by day 5 exposure to CSE Elk 1 mRNA levels were reducing and by day 7 Elk1 mRNA levels were lower than controls (Fig. 6A).

Western blot of lysates obtained from iHBECs exposed to $3 \%$ CSE led to reduced ELK1 after 7 days of exposure, which was confirmed by densitometric analysis (Fig. 6B).

To confirm the results of in vitro cell cultures, precision cut lung slices (PCLS) obtained from uninjured lungs from wild-type mice were exposed to either 0 or $3 \%$ CSE for 7 days. Levels of ELK1 protein reduced by $50 \%$ in PCLS exposed to $3 \%$ CSE (Fig. 6C).

\section{Discussion}

IPF specifically, and fibrotic diseases more generally, affect primarily the elderly and have been associated with age-related processes such as telomere shortening (Cronkhite et al., 2008; Armanios et al., 2007; Alder et al., 2008). We have previously identified a repressive role for the transcription factor ELK1 in the pathogenesis of IPF and have demonstrated that mice without ELK1 have an exaggerated fibrotic response to lung injury through loss of repression of $\alpha v \beta 6$ integrin mediated TGF $\beta$-activation (Uhlén et al., 2015). We therefore hypothesized that if the ELK1 transcriptional repression was important for the regulation of homeostatic functions of the $\alpha v \beta 6$ integrin then mice with global deficiency of $E l k 1$ would develop age-related fibrotic changes.

We assessed the phenotype of $E l k 1^{-/ O}$ mice aged for 12 months without injury and found that these mice developed features consistent with pulmonary fibrosis despite the absence of injury. This is unusual in many genetic models of lung fibrosis which often require a second "hit" to promote fibrosis (Moore et al., 2013), although this may be due to the small number of studies that have looked at the effect of ageing in genetically modified mice. Some age-related studies include Relaxin ${ }^{-/}$ mice have been shown to develop features consistent with small airway fibrosis and renal fibrosis (Samuel et al., 2003, 2004). Furthermore, specific deletion of the telomere shelterin protein TRF1 also leads to age dependent lung fibrosis (Naikawadi et al., 2016), as well as, mutations in genes with causal associations with IPF (Cheng et al., 2005; Blackwell, 2018). It is therefore apparent that genetic changes can promote spontaneous fibrosis across a range of organs. 
It is interesting to note that the fibrotic phenotype in ELK1 deficient animals did not map onto the organs with the highest levels of ELK1 in wild-type animals. Although these data did not show a significant increase in Itgb6 mRNA in whole lung lysates from aged Elk1 $1^{-/ 0}$ mice, the direction and magnitude of change were similar our previous studies which identified that loss of ELK1 promotes lung fibrosis through enhanced expression of the epithelial specific $\beta 6$ integrin subunit Tatler et al., 2016. This may explain why no phenotype relating to cardiac fibrosis was observed as epithelial injury and $\alpha v \beta 6$ integrins are not primarily involved in fibrogenesis of the heart, where other $\alpha v$ integrins are involved (Murray et al., 2017).

Loss of ELK-1 also lead to mild liver fibrosis as assessed by Sirius red staining, although there was no increase in liver hydroxyproline levels. This may reflect the mild nature of the fibrosis, possibly below the level of sensitivity for this assay, and the fact that fatty liver was the major phenotype observed. Within the liver it is possible that ELK1 may regulate $\alpha v \beta 6$ expression, although loss of the $\beta 6$ integrin subunit did not protect against $\mathrm{CCl} 4$ induced hepatic fibrosis (Henderson et al., 2013) and levels of Itgb6 mRNA were undetectable in whole lung lysates. It is interesting to note that despite the presence of considerably more fat in the livers of $E l k 1^{-/ O}$ mice compared with wild-types, there was no difference in their body weight growth curves over the 1-year period (Supplemental Fig. 4) suggesting that ELK1 has a direct heptatoprotective effect. We cannot exclude that cell specific loss of ELK1 mediated repression of $\alpha v \beta 6$ integrins may be involved in the observed phenotype, and indeed loss or inhibition of the $\alpha v \beta 6$ integrin has been shown to protect against biliary fibrosis and biliary cells make up only a small proportion of the total liver cell population and it is therefore likely that any increase in cholangiocyte $\alpha v \beta 6$ expression was not observed in the whole liver lysates used in this study (Popov et al., 2008; Wang et al., 2007). Previous studies have suggested that the $\alpha v \beta 1$ integrin is a profibrotic integrin in both the lung and liver (Henderson et al., 2013), however we consider it unlikely that ELK1 is mediating protective affects via Itgb1 as loss of ELK lead to a reduced Itgb1 in the lung and was unchanged in the liver, although again we can't exclude cell specific effects.

Intriguingly, kidneys from $E l k 1^{-/ O}$ mice did not show any fibrotic phenotype despite a well-established role of the $\alpha v \beta 6$ integrin in renal fibrosis. Ischaemia reperfusion injury in rat kidneys promotes $\alpha \mathrm{v} \beta 6$ expression and TGF $\beta$ activation (Geng et al., 2012). In a UUO model of renal fibrosis, mice are partially protected by loss of $\beta 6$ integrin and similarly Alport mice are protected from fibrosis by administration of a $\beta 6$ blocking antibody (Hahm et al., 2007; Ma et al., 2003). However, it is notable that loss of ELK1 in the kidneys had a profound effect on reducing Itgb6 expression in the kidneys suggesting that in this organ ELK1 acts a transcriptional activator of Itgb6, rather than as a repressor. It is known that post-transcriptional modifications of ELK1 can affect its function (Chung et al., 1998; Yang et al., 2003; Yang and Sharrocks, 2004). We hypothesise that exposure to renal specific substrates may promote a distinct post-transcriptional profile, such as an excess of sumoylated ELK1, in the renal tract compared with the lung.

The role of the $\alpha v \beta 1$ integrin in renal fibrosis is highly cell specific with deletion of $\beta 1$ in collecting duct principle cells promoting fibrosis (Mamuya et al., 2017) but, loss of fibroblast $\alpha v \beta 1$ integrins ameliorates it (Henderson et al., 2013; Chang et al., 2017). The data presented here show a mild and insignificant increase in Itgb1 mRNA expression which may explain the lack of fibrosis in this aged model. However, levels of Itgb1 mRNA may not truly reflect cell surface expression of $\alpha v \beta 1$ integrins as $\beta 1$ integrin subunits are produced in excess within a cell and it is expression levels of the $\alpha$ subunit that governs expression for $\beta 1$ containing heterodimers. Unlike the lung and the liver, Itgb5 is increased in the kidneys of $E l k 1^{-/ O}$ mice at the message level compared to controls and increased myofibroblast expansion has been associated with increased $\alpha v \beta 5$ levels in the kidney (McCurley et al., 2017). It is clear from our studies that there are cell and organ specific effects of ELK1 on the regulation of the different integrin subunits which are likely to determine the fibrotic phenotype associated with aging.

This study provides detailed phenotyping of uninjured age-related fibrosis in $E l k 1^{-/ 0}$ mice, including the identification of ELK1 as an agedependent mediator of lung and liver fibrosis in male C57BL/6 mice. Both lung and liver fibrosis occur in older people and this is especially the case for patients with idiopathic pulmonary fibrosis where there is no clearly identifiable aetiological agent. It is not clear how ELK1 may be lost over time to promote disease and our data suggest that ageing itself is not sufficient to cause a loss of Elk1 expression. Genetic studies have not identified an association with ELK-1, or indeed polymorphisms in the $\mathrm{X}$ chromosome and pulmonary fibrosis, similarly no changes have been found associating the ELK-1 epigenome and IPF. These data suggest that cigarette smoke can, in principle, reduce ELK1 expression. Therefore, we hypothesise that cigarette smoking may reduce ELK1 which may over time may promote fibrosis.

As the Elk1 gene is located on the $\mathrm{X}$ chromosome, there are particular benefits to using only male mice in these studies including eliminating the effects of female hormones on the fibrotic response (Han et al., 2008). Whilst ageing the mice beyond one year may have further exaggerated the age-related fibrotic effects and given more dramatic phenotypes it is reassuring that a phenotype was observed after only 12 months. A further strength is the application of a known fibrosis risk factor on both a cell line and physiologically viable PCLS both demonstrating a link between smoking and the loss of ELK1. These studies do however have some limitations. Most notably the use of immortalised epithelial cells may not recapitulate the response of primary alveolar epithelial cells. Furthermore, due to the breeding strategy required for the gene deletion of ELK1 on the X chromosome these studies were limited to males and therefore the biological effect of sex on the development of fibrosis cannot be assessed.

In conclusion, our data support a role for the loss of ELK1 in promoting age-related fibrosis in both the lung and liver. These data also highlight the organ-specific effects of ELK1, including a differential effect on Itgb6 in the lung and kidney, where ELK1 appears to act as a transcriptional activator of Itgb6 gene expression. Finally, exposure to known fibrogenic risk factors can reduce expression of ELK1 in the lung epithelium, which may in part explain how cigarette smoke increases the risk of IPF. These findings highlight the importance of normal functioning ELK1 in protecting two major organs from age related fibrosis.

\section{Author contributions}

GJ and ALT conceived, designed, and supervised the study. JTC, $\mathrm{AH}, \mathrm{FO}, \mathrm{CW}, \mathrm{CJ}, \mathrm{AEJ}$ and JL performed experiments. AN and SA were involved with studies using $E l k 1^{-/ O}$ mice. JTC and IDS performed data analyses. JTC, BB, FO and KS provided histopathological assessment. JTC, AH and RCEP facilitated collection and processing of mouse tissues. JTC, GJ and ALT drafted the manuscript. All authors provided comments on the manuscript and had final approval of the submitted version.

\section{Conflicts of interest}

Nothing declared.

\section{Acknowledgements}

We would like to acknowledge the Nuffield Foundation and the two placement students, Miss Zainab Mubashra and Miss Gurleen Virk, whose input has supported this project. Also, we would like to thank Miss Rachel Walker, her efforts as a BSc student have aided this work. Finally, we thank the Nottingham Health Science Biobank (NHSB) for help with tissue processing and the School of Life Sciences IMaging (SLIM) for their microscopy expertise. Medical Resaerch Foundation grant (ALT)MRF-091-0004-RG-TATLE. 


\section{Appendix A. Supplementary data}

Supplementary material related to this article can be found, in the online version, at doi:https://doi.org/10.1016/j.biocel.2019.105668.

\section{References}

Wynn, T.A., 2008. Cellular and molecular mechanisms of fibrosis. J. Pathol. Weiskirchen, R., Weiskirchen, S., Tacke, F., 2019. Organ and tissue fibrosis: molecular signals, cellular mechanisms and translational implications. Mol. Aspects Med.

Henderson, N.C., Arnold, T.D., Katamura, Y., et al., 2013. Targeting of av integrin identifies a core molecular pathway that regulates fibrosis in several organs. Nat. Med.

Tatler, A.L., Jenkins, G., 2012. TGF-beta activation and lung fibrosis. Proc. Am. Thorac. Soc.

Popov, Y., Patsenker, E., Stickel, F., et al., 2008. Integrin $\alpha v \beta 6$ is a marker of the progression of biliary and portal liver fibrosis and a novel target for antifibrotic therapies. J. Hepatol,

Häkkinen, L., Koivisto, L., Gardner, H., et al., 2004. Increased expression of $\beta 6$-Integrin in skin leads to spontaneous development of chronic wounds. Am. J. Pathol.

Hahm, K., Lukashev, M.E., Luo, Y., et al., 2007. avß6 integrin regulates renal fibrosis and inflammation in alport mouse. Am. J. Pathol.

Reed, N.I., Jo, H., Chen, C., et al., 2015. The $\alpha v \beta 1$ integrin plays a critical in vivo role in tissue fibrosis. Sci. Transl. Med.

Chang, Y., Lau, W.L., Jo, H., et al., 2017. Pharmacologic blockade of $\alpha$ v $\beta 1$ integrin ameliorates renal failure and fibrosis in vivo. J. Am. Soc. Nephrol.

Sharrocks, A.D., 2001. The ETS-domain transcription factor family. Nat. Rev. Mol. Cell Biol.

Uhlén, M., Fagerberg, L., Hallström, B.M., et al., 2015. Tissue-based map of the human proteome. Science (80-).

Tatler, A.L., Habgood, A., Porte, J., et al., 2016. Reduced ets domain-containing protein Elk1 promotes pulmonary fibrosis via increased integrin avß6 expression. J. Biol. Chem. 291, 9540-9553.

Cesari, F., Rennekampff, V., Vintersten, K., et al., 2004. Elk-1 knock-out mice engineered by flp recombinase-mediated cassette exchange. Genesis.

Brunt, E.M., Janney, C.G., Di Bisceglie, A.M., et al., 1999. Nonalcoholic steatohepatitis: a proposal for grading and staging the histological lesions. Am. J. Gastroenterol.

Cronkhite, J.T., Xing, C., Raghu, G., et al., 2008. Telomere shortening in familial and sporadic pulmonary fibrosis. Am. J. Respir. Crit. Care Med.

Armanios, M.Y., JJ-L, C., Cogan, J.D., et al., 2007. Telomerase mutations in families with idiopathic pulmonary fibrosis. N. Engl. J. Med.

Alder, J.K., JJ-L, C., Lancaster, L., et al., 2008. Short telomeres are a risk factor for idiopathic pulmonary fibrosis. Proc. Natl. Acad. Sci. U. S. A.

Moore, B.B., Lawson, W.E., Oury, T.D., et al., 2013. Animal models of fibrotic lung disease. Am. J. Respir. Cell Mol. Biol.

Samuel, C.S., Zhao, C., Bathgate, R.A.D., et al., 2003. Relaxin deficiency in mice is associated with an age-related progression of pulmonary fibrosis. FASEB J.

Samuel, C.S., Zhao, C., Bond, C.P., et al., 2004. Relaxin-1-deficient mice develop an agerelated progression of renal fibrosis. Kidney Int.

Naikawadi, R.P., Disayabutr, S., Mallavia, B., et al., 2016. Telomere dysfunction in alveolar epithelial cells causes lung remodeling and fibrosis. JCI Insight.

Cheng, Q., Zhao, Y., Lawson, W.E., et al., 2005. The effects of intrinsic pathway protease deficiencies on plasminogen-deficient mice. Blood.

Blackwell, T.S., 2018. Lung injury and fibrosis induced by a mutant form of surfactant protein C. J. Clin. Invest.

Murray, I.R., Gonzalez, Z.N., Baily, J., et al., 2017. av integrins on mesenchymal cells regulate skeletal and cardiac muscle fibrosis. Nat. Commun.

Wang, B., Dolinski, B.M., Kikuchi, N., et al., 2007. Role of $\alpha v \beta 6$ integrin in acute biliary fibrosis. Hepatology.

Geng, H., Lan, R., Singha, P.K., et al., 2012. Lysophosphatidic acid increases proximal tubule cell secretion of profibrotic cytokines pdgf-b and ctgf through lpa2- and gaqmediated rho and $\alpha \mathrm{v} \beta 6$ integrin-dependent activation of tgf- $\beta$. Am. J. Pathol.

Ma, L.-J., Yang, H., Gaspert, A., et al., 2003. Transforming growth Factor- $\beta$-dependent and -independent pathways of induction of tubulointerstitial fibrosis in $\beta 6-/-$ mice. Am. J. Pathol. 163, 1261-1273.

Chung, K.-C., Gomes, I., Wang, D., et al., 1998. Raf and Fibroblast Growth Factor Phosphorylate Elk1 and Activate the Serum Response Element of the Immediate Early Gene pip92 by Mitogen-Activated Protein Kinase-Independent as Well as -Dependent Signaling Pathways. Mol. Cell. Biol. 18, 2272-2281.

Yang, S.H., Jaffray, E., Hay, R.T., et al., 2003. Dynamic interplay of the SUMO and ERK pathways in regulating Elk-1 transcriptional activity. Mol. Cell.

Yang, S.H., Sharrocks, A.D., 2004. SUMO promotes HDAC-mediated transcriptional repression. Mol. Cell.

Mamuya, F.A., Xie, D., Lei, L., et al., 2017. Deletion of $\beta 1$-integrin in collecting duct principal cells leads to tubular injury and renal medullary fibrosis. Am. J. Renal Physiol.

McCurley, A., Alimperti, S., Campos-Bilderback, S.B., et al., 2017. Inhibition of $\alpha \mathrm{v}$ B 5 integrin attenuates vascular permeability and protects against renal ischemia-reperfusion injury. J. Am. Soc. Nephrol.

Han, M.K., Murray, S., Fell, C.D., et al., 2008. Sex differences in physiological progression of idiopathic pulmonary fibrosis. Eur. Respir. J. 31, 1183-1188. 